\title{
Study of structured water and its biological effects
}

\begin{abstract}
Background: The study of water structuring under the influence of different devices and biological effects of such waters require the development of different methods.

Methods: The Dynamic Electrophotonic Imaging (EPI) Analysis based on Gas Discharge Visualization (GDV) method was used to study the structured water, as well as its influence to biological substances: food samples and sprouts.

Results: Statistically significant difference between control and experimental samples were demonstrated on three brands of waters, but no effect on the fourth water brand. For samples of food products: apple, banana, carrot, celery, potato, cheese and sausage statistically significant difference between control and experimental samples was found. Observation of the germination rate of rye and oat seeds showed that the appearance of the first root (seed pecking) occurred 1 day earlier in the experiment than in the control. In the experiment, an average of $81.5 \%$ to $57 \%$ of seeds grew, while in the control $47.8 \%$. In the experiment there were $63.2 \%$ to $54.0 \%$ of the right seedlings, while in the control $47.8 \%$. The development of potato roots in the experimental variants was about 2 times higher than the roots in the control variants.
\end{abstract}

Conclusions: Structuring of water under the influence of Amezcua BioDisc3 by Qnet had a significant effect both on EPI/GDV parameters of water and different biological substances influenced by this disc. This opens up wide prospects for the practical implementation of the EPI/GDV method.

Keywords: water structuring, biological effects, electrophotonic imaging, gas discharge visualization, amezcua bio disc 3
Volume 12 Issue $5-2019$

\author{
Konstantin Korotkov $v^{1,2}$ \\ 'Federal State Budget Institution, Saint-Petersburg Scientific \\ ${ }^{2}$ Research Institute for Physical Culture, Russia
}

Correspondence: Federal State Budget Institution, SaintPetersburg Scientific Research Institute for Physical Culture, Russia, Email korotkov2000@gmail.com

Received: September 09, 2019 | Published: October 07, 2019

\section{Introduction}

Currently, considerable attention is being focused on the study of the structural properties of water and the possibility of data transfer through water. A lot of controversial information we may find concerning the memory of water. ${ }^{1}$ According to the viewpoint that has shaped, the phenomena observed during the experiments are determined by the processes of clusters and clathrates formation, mainly at the atoms of admixtures. ${ }^{2}$ The task of introducing these notions into the scope of contemporary scientific thinking requires, first of all, a set of probative and reproducible experimental facts. Water is a complex subject of study, and its properties depend on a great number of factors; this requires that several independent techniques should be used in parallel and that new informative methods for the study of water properties should be developed and introduced into practice. ${ }^{3,4}$ From the theoretical principles based on quantum electrodynamics. ${ }^{5}$ follows that liquid water should be a multiphase, non-equilibrium and, therefore, the active complex system. That alone makes water a complex dynamic system with much richer behavior than that of any homogenous matter. For example, under certain conditions, it should change its state in response to the weak resonance signals, and also for a long time maintain such a condition. This property is known as "structured water". ${ }^{1,6}$ In the last decades, convincing experimental evidence has appeared, supporting the theoretical model of Italian physicists. It was shown, for example, that a sufficient condition for the emergence of a long-living organized phase in aqueous systems is the presence of the interface of liquid water with hydrophilic surfaces with which it contacts, and even the interfaces of water with air. ${ }^{7}$ The Professor of the University of Washington Gerald Pollack received this evidence. ${ }^{8}$ He has developed a simple experimental model, using which only in a few years had made a number of discoveries showing how complex is even very clean water. All these conditions promote water separation into two different substances (phases). Interaction of these phases having very different properties is the cause of many phenomena. This is closely related to the special properties of water in living matter: in particular, in the cell protoplasm. Having a long history, is the idea that water in living cells, filled with proteins, polysaccharides, and nucleic acids, must be structured due to the interaction with all these particles and so must differ in its properties from "normal" water. Since the beginning of the last century, many prominent physiologists, biochemists, chemists, and cytologists have defended this idea. They proceeded from the obvious to any unbiased scholar facts, and thus formed the basis of colloid chemistry. ${ }^{9}$ The different states of water in the cellular and extra-cellular phases prevent these two phases from mixing (and therefore, this concept is called the phase theory of protoplasm). Water in protoplasm differs in solvent capacity from the external water and the uneven distribution of substances between cell and environment should be explained - not by the presence of specific pumps and channels in a hypothetical semi-permeable membrane that separates cell from the environment - but by different distribution coefficients between the two aqueous phases. ${ }^{11,12}$ The water in erythrocytes possesses a quasi-crystalline structure, and the molecules form a complex spatial network whose loops contain hemoglobin molecules. Appropriate calculations showed, that in blood, nearly all the water occurs in the interfacial state.

The study of water structuring under the influence of different devices and biological effects of such waters requires the development of different methods. In this paper we are presenting results of the application of the Dynamic Electrophotonic Imaging (EPI) Analysis based on Gas Discharge Visualization (GDV) method to study the structured water, as well as its influence to biological substances: food samples and sprouts. There are a lot of evidence that drinking structured water is beneficial for health. ${ }^{13-15}$ but not enough published research papers. This pilot study was conducted to check the validity of these claims. 


\section{Materials and methods}

Study of Electrophotonic parameters of liquids is based on using a commercially produced instrument "Bio-Well", which is manufactured by Bio-Well company (www.bio-well.com). This instrument is well-known for analyzing stimulated photon emission from human fingers which are being used for health and well-being analysis, ${ }^{17-22}$ analysis of athletes,${ }^{23}$ environment, ${ }^{24}$ and water. ${ }^{25,26}$ When the EPI parameters are measured for liquid subjects, a drop of the liquid is suspended at $2-3 \mathrm{~mm}$ distance above the glass surface of the optical window of the device, and the glow from the meniscus of the liquid is registered (Figure 1).

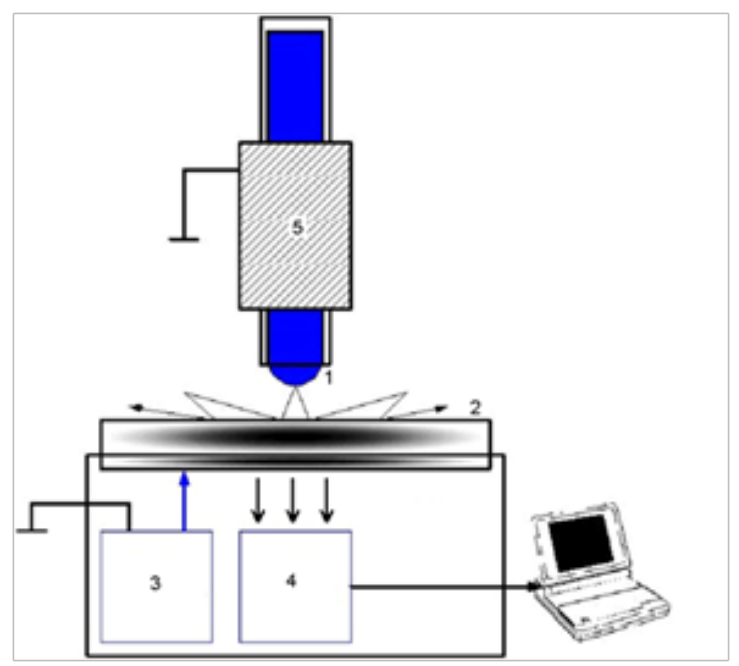

Figure I Principle of water measuring with EPI device. I -Water drop; 2 - gas discharge; 3 - power unit; 4 - optical system; 5 - electrode.

The volume of liquid is about $5 * 10^{-3} \mathrm{ml}$. The train of triangular bipolar electrical $10 \mathrm{mcs}$ impulses of amplitude $3 \mathrm{kV}$ at a steep rate of $106 \mathrm{~V} / \mathrm{s}$ and a repetition frequency of $103 \mathrm{~Hz}$ is applied to the conductive transparent layer at the backside of the quartz electrode thus generating electromagnetic field at the surface of the electrode and around the drop. Under the influence of this field, the drop produces a burst of electron-ion emission and optical radiation light quanta in the visual and ultraviolet light regions of the electromagnetic spectrum. These particles and ions initiate electron-ion avalanches, which give rise to the sliding gas discharge along the dielectric surface. ${ }^{26}$ Spatial distribution of discharge channels is registered through a glass electrode by the optical system with a charge coupled device TV camera, and then it is digitized in the computer.

For structuring water, Amezcua Bio Disc 3, an energy wellness glass disc by QNet was used. This glass disc with a diameter of 3.5 inches $(9 \mathrm{~cm})$ and 0.4 inches $(1 \mathrm{~cm})$ thick is made up of a special combination of natural granulated crystals, energized using their very own proprietary process technology and has a masterful geometry design that creates a field of essential energy-balancing qualities and optimally balance the body's interactions with environmental stressors. The Amezcua Bio Disc 3 by QNet is similar to the glass being used in solid-state lasers. It transforms the light flux falling on it into quasi-coherent radiation. Water in a transparent jar placed on top of the Amezcua Bio Disc 3 becomes structured after 10minutes and continues to increase in structure, as it has been shown by physical experiments.

\section{Results}

\section{Study of structured water by EPI method}

4 different types of commercially available natural water, 2 bottles each, were used for experiments. Bottles were kept closed in dark cool place. 4 bottles were positioned on structuring device, other bottles served as control. Each time 5 probes of $1 \mathrm{ml}$ water were tested 10 times, 400 tests overall each time. Testing was done:

a. Immediately after structuring.

b. After 3hours.

c. Day 2

d. Day 3

Results of the study are presented in Table 1. As we see from these data, it was statistically a significant influence on three brands of water, and no effect on the fourth brand of water. No correlations with mineral content were found.

Table I EPI parameters of structured and control waters

\begin{tabular}{llll}
\hline Test & $\begin{array}{l}\text { Control, } \\
\text { averaged on } \\
\mathbf{5 0} \text { tests }\end{array}$ & $\begin{array}{l}\text { Structured, } \\
\text { averaged on } \\
\mathbf{5 0} \text { tests }\end{array}$ & $\begin{array}{l}\text { Student } \\
\text { test } \mathbf{p} \\
\text { value }\end{array}$ \\
\hline Bottle I Bon Aqua & & & \\
\hline Immediately after & $848+/-102$ & $1204+/-184$ & $<0.000 \mid$ \\
3 hours & $830+/-249$ & $1028+/-314$ & 0.05 \\
Day 2 & $1216+/-365$ & $1 \mid 13+/-337$ & $<0.000 \mid$ \\
Day 3 & $1319+/-396$ & $1156+/-347$ & $<0.000 \mid$
\end{tabular}

Bottle 2 Evian

\begin{tabular}{llll}
\hline Immediately after & $1154+/-347$ & $1045+/-314$ & $<0.0001$ \\
3 hours & $1756+/-526$ & $1429+/-409$ & $<0.0001$ \\
Day 2 & $1142+/-346$ & $\mid 1561+/-468$ & $<0.0001$ \\
Day 3 & $1849+/-523$ & $1775+/-533$ & 0.001
\end{tabular}

Bottle 3 Istochnik

\begin{tabular}{llll}
\hline Immediately after & $1349+.-147$ & $1336+.-336$ & 0.64 \\
3 hours & $2099+.-629$ & $2064+.-619$ & 0.24 \\
Day 2 & $2179+.-634$ & $2218+.-617$ & 0.28 \\
Day 3 & $2371+.-708$ & $2202+.-666$ & 0.12 \\
Bottle 4 Nalchik & & & \\
\hline Immediately after & $1414+/-356$ & $1619+/-25 \mid$ & $<0.0001$ \\
3 hours & $1818+/-85.4$ & $1504+/-208$ & $<0.0001$ \\
Day 2 & $2613+/-785$ & $2213+/-666$ & $<0.0001$ \\
Day 3 & $2742+/-822$ & $2587+/-777$ & $<0.0001$
\end{tabular}


At the same time, the $\mathrm{pH}$ and conductivity were also measured for all water samples. No significant changes were noted.

\section{Study of the influence of structured water on biological substances}

Food samples: Fresh products purchased from the retail store were used. For all food types 10 equal samples of $10 \mathrm{~mm}$ in diameter and $5 \mathrm{~mm}$ thickness were cut with a cutter. For sausage 10 slides of equal thickness were cut from the middle part of the product. 5 samples were left at the lab table, while 5 samples were positioned on the structuring device for 15 minutes. 50 readings for every sample were done, which allowed having normal distribution of the data. Results of the study are presented in Table 2. As we see from these data, in all studied samples it was a statistically significant difference between control and experimental samples. No change in glow parameters was noted in the use of dried beet and carrot samples.

Seed and sprouts: Rye and oat seeds were used as objects of research. The seeds were calibrated before the experiment, placed by 50 seeds in Petri dishes on filter paper, and then $7 \mathrm{ml}$ of water of the "Holy spring" brand was added to each Petri dish. Experimental Petri dishes were positioned on structuring device. In every test were
2 experimental and 2 control dishes. Observations were made daily, noting the appearance of roots on the 3rd day and seedlings on the 4th day. It was known that in normal conditions cereal plants have left and right-swirled sprouts in a ratio of 1:1, while it the influence of structured water leads to an increase in the number of right-swirled plants that have increased productivity. The results are presented in Table 3. It was shown that in the experimental variants there was a significant and reliable $(\mathrm{p}<0.001)$ increase in the right seedlings, indicating a favorable effect of Amezcua Bio Disc 3 on water and swollen seeds in it. Observation of the germination rate showed that the appearance of the first root (seed pecking) occurred 1 day earlier in the experiment than in the control. In the experiment, an average of $81.5 \%$ to $57 \%$ of seeds grew, while in the control $47.8 \%$. In the experiment, there were $63.2 \%$ to $54.0 \%$ of the right seedlings, in the control $47.8 \%$

Single stem potatoes were divided into pieces (4 Experiment and 4 Control) by culturing them in test tubes with a nutrient medium (Marafie-Skogo). The result was estimated at 7-8days according to the degree of root system development. The development of roots in the experimental variants was about 2 times higher than the roots in the control variants. The results are presented in Table 4.

Table 2 EPI parameters of food samples

\begin{tabular}{llll}
\hline Sample & Control & Structured & p value \\
\hline Apple & $50073+/-1500$ & $51164+/-1800$ & 0.01 \\
Banana & $25448+/-1200$ & $25401+/-787$ & 0.62 \\
Carrot & $90434+/-778$ & $93195+/-434$ & $<0.001$ \\
Celery & $89156+/-844$ & $88288+/-743$ & 0.02 \\
Potato & $89780+/-626$ & $89092+/-606$ & 0.02 \\
Cheese & $23705+/-401$ & $22759+/-543$ & $<0.001$ \\
Sausage & $24704+/-607$ & $25411+/-393$ & $<0.001$ \\
\hline
\end{tabular}

Table 3 Evaluation of structuring device influence on the sprouting of rye

\begin{tabular}{|c|c|c|c|c|c|c|c|}
\hline \multirow{2}{*}{ Variant } & \multirow{2}{*}{ Amount of seeds } & \multicolumn{2}{|c|}{ Sprouted } & \multicolumn{2}{|c|}{ Right sprouts } & \multicolumn{2}{|c|}{ Left sprouts } \\
\hline & & seeds & $\%$ & seeds & $\%$ & seeds & $\%$ \\
\hline К- I & 50 & 23 & 46,0 & 11 & 47,8 & 12 & 52.2 \\
\hline K-2 & 50 & 22 & 44,0 & 10 & 45,5 & 12 & 54.5 \\
\hline K-3 & 50 & 25 & 50,0 & 12 & 48,0 & 13 & 52.0 \\
\hline K-4 & 50 & 24 & 48,0 & 12 & 59,0 & 12 & 50.0 \\
\hline \multicolumn{2}{|l|}{ Control } & \multicolumn{2}{|c|}{ Mcp $=47,0 \%$} & \multicolumn{2}{|c|}{ Mcp $=47,8 \%$} & \multicolumn{2}{|c|}{$\mathrm{Mcp}=52.2 \%$} \\
\hline E-I & 50 & 22 & 44.0 & 14 & 63.6 & 8 & 36.4 \\
\hline $\mathrm{E}-2$ & 50 & 30 & 60.0 & 19 & 63.3 & 11 & 36.7 \\
\hline$E-3$ & 50 & 33 & 66.0 & 21 & 63.6 & 12 & 36.4 \\
\hline E-4 & 50 & 29 & 58.0 & 18 & 62.1 & 11 & 37.9 \\
\hline Experiment & & $M c p=5$ & $0, \%$ & $M c p=6$ & & Mcp $=$ & $9, \%$ \\
\hline
\end{tabular}


Table 4 Evaluation of structuring device influence on the germination of cuttings

\begin{tabular}{lllllllll}
\hline Variant & E-I & E-2 & E-3 & E-4 & K-I & K-2 & K-3 & K-4 \\
\hline Size of roots, $\mathrm{mm}$ & $\mathrm{I} 5+/-4$ & $\mathrm{I} 6+/-3$ & $\mathrm{I} 7+/-4$ & $\mathrm{I} 6+/-4$ & $5+/-2$ & $3+/-2$ & $6+/-2$ & $5+/-2$ \\
\hline
\end{tabular}

\section{Discussion}

On the Internet you can find a wide range of devices, the authors of which claim that they are designed to structure water. However, almost none of them provide the results of studies confirming their claims. The EPI/GDV method offers a relatively simple, affordable technology for checking the structuring of water under the influence of different devices and the effects of this water on biological subjects and human health. The data presented in this paper allow several generalizations to be made:

a. The structuring effect depends on the initial composition of the water: of the four studied brand of waters, one did not show any effect under the influence of the structuring device.

b. After opening a sealed bottle of water EPI/GDV parameters increase with time, both in the source water and after the device impact. This indicates the interaction of water with the environment, and this issue requires further study.

At the interaction of the structuring device with foodstuffs, the structuring of the liquid in the products takes place, which is reflected in the EPI/GDV indicators. This effect is not observed with dry products.

It is expedient to use several methods of water research in parallel to obtain complex information.

In the pilot study ${ }^{27}$ beneficial effect for 15 people of 30-day consumption of 1 liter of water structured with the help of Amezcua Bio disc 3 on the composition of the body and blood, a significant decrease in creatinine levels, as well as an increase in glomerular filtration of the kidneys, which reflects the improvement of renal excretory function and a decrease in the level of endogenous intoxication of the body; the growth of HRV and Bio-Well GDV parameters reflecting parasympathetic activity and variability of heart rhythm and the decrease of parameters which reflect the degree of sympathetic regulation and centralization of heart rhythm. No such changes were observed in the control group of 15 people that consumed unstructured water of the same brand.

\section{Conclusions}

Structuring of water under the influence of various devices and the impact of this water on biological objects can be identified by the EPI/GDV method. This opens up wide prospects for practical implementation of the method.

\section{Acknowledgments}

None.

\section{Conflicts of interest}

Author declares there are no conflicts of interest towards this article

\section{Funding}

This research received no external funding.

\section{References}

1. Johansson B. Effects of Functional Water on Heart Rate, Heart Rate Variability, and Salivary Immunoglobulin A in Healthy Humans: A Pilot Study. J Altern Complement Med. 2009;15(8):871-877.

2. Del Giudice E, Vitiello G. Role of the electromagnetic field in the formation of domains in the process of symmetry-breaking phase transition. Phys Rev A. 2006;74:1-9.

3. Voeikov VL, Del Giudice E. Water Respiration - The Basis of the Living State. Water. 2009;1(1):52-75.

4. Voeikov VL, Korotkov KG. The Emerging Science of Water. USA: Amazon; 2017.

5. Del Giudice E, Preparata G, Vitiello G. Water as a free electric dipole laser. Phys Rev Lett. 1988;61(9):1085-1088.

6. Clark GNI, Cappa CD, Smith JD, et al. The structure of ambient water. Molecular Phys. 2010;108(11):1415-1433.

7. Tokushima T, Harada Y, Takahashi O, et al. High resolution Xray emission spectroscopy of liquid water: The observation of two structural motifs. Chem Phys Lett. 2008;460(4-6):387-400.

8. Chen CS, Chung WJ, Hsu IC, et al. Force field measurements within the exclusion zone of water. J Biol Phys. 2012;38(1):113-120.

9. Pollack GH. The Fourth Phase of Water. EDGESCIENC. 2013.

10. Henry M. The state of water in living systems: from the liquid to the jellyfish. Cell Mol Biol (Noisy-le-grand). 2005;51(7):677-702.

11. Nasonov DN. Protoplasm reactions. Moscow. 1959.

12. Ling, Gilbert. The Physical State of Water in Living Cell and Model Systems. Annals of the New York Academy of Sciences. 2006;125(2):401-417.

13. Ho L, Thas O, Echelpoel WV, Goethals P. A Practical Protocol for the Experimental Design of Comparative Studies on Water Treatment. Water. 2019;11(1):162.

14. Jillian Levy. Structured Water: The Next Best Treatment for Muscular, Skin \& Mood Disorders? Axe. 2015.

15. https://www.alkalinewaterplus.com/doctors-experts/

16. http://www.crystalblueent.com/testimonials - results.html

17. Korobka IE, Yakovleva TG. Electrophotonic Imaging technology in the diagnosis of autonomic nervous system in patients with arterial hypertension. J Appl Biotechnol Bioeng. 2018;5(1):112-116.

18. Korotkov K. Review of EPI papers on medicine and psychophysiology published in 2008-2018. Int $J$ Complement Alt Med. 2018;11(5):311-315.

19. Bhat RK, Deo G, Mavathur R, et al. Correlation of Electrophotonic Imaging Parameters with Fasting Blood Sugar in Normal, Prediabetic, and Diabetic Study Participants. J Evid Based Complementary Altern Med. 2017;22(3):441-448.

20. Yakovleva EG, Korotkov KG, Fedorov ED, et al. Engineering Approach To Identifying Patients With Colon Tumors On The Basis Of Electrophotonic Imaging Technique Data. Open Biomed Eng J. 2016;10:72-80. 
21. Yakovleva EG, Buntseva OA, Belonosov SS, et al. Identifying Patients with Colon Neoplasias with Gas Discharge Visualization Technique. $J$ Altern Complement Med. 2015;21(11):720-724.

22. Aleksandrova EV, Kovelkova TN. Electrophotonic Analysis of Arterial Hypertension. J of Science of Healing Outcome. 2015;7(28):4-12.

23. Drozdovski A, Gromova I, Korotkov K, et al. Express-evaluation of the psycho-physiological condition of Paralympic athletes. Open Access J Sports Med. 2012;3:215-222.

24. Rubik B, Jabs H. Bio-Well Sputnik Assessment of Changes in the Energy Field During the August 21, 2017 Solar Eclipse. Frontier Science Journal. 2017;1-4.
25. Korotkov K, Orlov D. Analysis of Stimulated Electrophotonic Glow of Liquids. Water. 2010;2(12);12-16.

26. Korotkov, K.; Korotkin, D. Concentration dependence of gas discharge around drops of inorganic electrolytes. J Appl Phys 2001;89:473.

27. Korotkov KG, Churganov OA, Gavrilova EA, et al. Influence of drinking structured water to human psychophysiology. J Appl Biotechnol Bioeng. 2019;6(4):171-177. 